\title{
Research on Image Line Drawing and Creative Graphic Design in Anyue Sculpture Carvings*
}

\author{
Kaimin Yang \\ Sichuan Conservatory of Music \\ Chengdu, China
}

\begin{abstract}
Anyue sculpture carvings' image line drawing art adopts the thought pattern of graphic creation and uses various creative means to highly summarize and convey the culture deposits and artistic appreciation of Anyue sculpture carvings. On the basis of artistic inheritance, image line drawing has a richer connotation and modern graphic design has more patterns of manifestations.
\end{abstract}

Keywords-Anyue sculpture carvings; image line drawing; creative graphic design; design study

\section{INTRODUCTION}

Anyue sculpture carvings refer to the abundant stone sculpture carving art in Anyue County, Sichuan. Anyue is called Puzhou in ancient times. "Puzhou . Topographical Advantages" in The Record of Scenic Spots Across the Country records that "Pu is famous for its beautiful stones", therefore the beauty of sculpture carving is called "Sculpture carving beauty". Mr. Wang Zhaowen, a famous aesthetician, praises sculpture carvings as being "antique, abundant, exquisite and beautiful". Anyue sculpture carvings have a long history, prospering from middle Tang Dynasty to Song Dynasty and Ming and Qing Dynasties.

Anyue sculpture carvings have great research value. Seen from current literature, there are 100 thousand stone sculptures or so, among which there are two sculptures above 15 meters tall. Among them, there is the whole body stone carving Reclining Buddha (Left reclining) which is of the greatest scale in Tang Dynasty, the Three Collections of Buddhist Scripture, the only one sculpture of this kind currently existing in China, the Water and Moon Kuan-Yin sculpture known as "The Orient Venus", the earlier sculpture of Esoteric Buddhism Liu Benzun's ten graphs of cultivation stories among the two such sculptures in China, carved stone sculptures of Buddha in Huayan cave praised as influential artistic works of sculpture, and sculptures of Sakya, Avalokitesvara cave, flying Apsaras and so on in Yuanjue Cave. Anyue sculpture carving art reflects the combination of ancient excellent native culture and imported culture. It "carries on the excellence of Dunhuang and provides guidance for Dazu" under the influence of sculpture carving art in northwestern China and Central Plains. Dazu sculpture carving carries on its modeling and it reflects ancient Anyue sculpture cravers' genius creation and artistic culture.

*“Research on Cultural Protection and Development of Anyue Sculpture Carvings" Project, Sichuan Provincial Department of Education 08SB084.

\section{IMAGE LINE DRAWING IN ANYUE SCULPTURE CARVINGS}

Since ancient times, Chinese people have been researching and exploring line drawing deeply. Art is connected and Chinese traditional art is of the same source. During the millennium, we discover the trace of line drawing art from Chinese painting, calligraphy, sculpture and sculpture caving. Anyue sculpture carving art boasts smooth, vivid and "dynamic and its figures boast rich and vivid lines, giving people a lifelike feeling. We can feel the charm of line drawing from Anyue sculpture carvings which are exquisite beyond compare left by successive dynasties. For example, sculptures of the three saints in Avatamsaka Sutra boast exquisite and elegant costume patterns and creases, and create the volume of body through ups and downs of lines in costume patterns, ribbons, waistbands and skirt hemline with smooth lines and elasticity, making the whole sculpture life-like and showing the craver's superb line drawing artistic beauty.

During the millennium development of art, Chinese traditional line drawing has high requirements for lines, reaching the degree of "Miscellaneous with one extra line and inadequate with one less line". Line drawing has independent aesthetic value beyond its modeling function. During the long development process of line drawing, image line drawing comes into being. "Yi" refers to "obtaining the meaning through careful observation of words" and "Image" refers to the object carrying the meaning. "Image" is borrowed and extended in meaning by various fields, referring to expression of the author's subjective emotion and feelings with objective images. Image line drawing summarizes, extracts, purifies and imagines objective images and expresses subjective images in line drawing modeling language through disbanding and reconstruction. Anyue sculpture carvings' image line drawing highly summarizes and shows its unique cultural deposits and aesthetic characteristic, boasting the beauty of special character and creativity. Take Thousand-Hand Kwan-yin Cave in Reclining Buddha Temple as an example, the creator doesn't realistically carve a thousand hands. Instead, the creator extracts and carves six hands with symbolic meanings. The hand with repeated line carving on surrounding palisades expresses the author's imagination of a thousand hands. The three-dimensional and plane hands correspond to each other with a stack-up sense consisting of repetition and a strong rhyme, making people astonished at the beauty in rich visual shock. 


\section{THE CHARACTERISTICS OF IMAGE LINE DRAWING IN THE NEW ERA}

During its development to nowadays, image line drawing inherits and carries forward the advantages of traditional line drawing, goes beyond the limitations of traditional realistic line drawing, restores and shows objective images, and creatively demonstrates subjective consciousness and subconsciousness. It artistically processes objective natural images beyond the limit of time and space and arranges images in different time and space and of different viewpoints in the same painting to express and carry the author's emotion. Image line drawing recombines elements through disbanding and reconstruction to finally achieve the ideal subjective image. Modern image line drawing demonstrates the subjective thought, creative thought and design thought with openness and compatibility. In the new era, image line drawing constantly intensifies design language to form new consciousness and creative means, absorbs and integrates the essence of various kinds of art and design, re-interprets and expands its connotation and denotation through adding modern design language and expands expression ways of other artistic designs at the same time.

\section{EXTENSIBILITY OF IMAGE LINE DRAWING IN CREATIVE GRAPHIC DESIGN}

The history of graphs can trace back to the ancient times. The cliff painting of archaeological discovery in caves of Raskerk, France has a history of over 10 thousand years, proving that people use graphs to demonstrate the world before appearance of written language. These graphs reflect people's psychological presentation activities at that time, showing that people at that time use these two-dimensional elements to express their feeling about and recognition of the objective world. Different from written language, graphs can go beyond the limit of language and intuitively convey information, reaching people's heart and arousing resonance among them. Modern graphs refer to visual signs to spread information and they need to be individualized, informationalized and signalized with creative thoughts. In the era of reading graphs, good graphs boast creativity with stronger visual impact than excellent and elegant documents. Holger Matthies, a German visual design master, says: "The excellence of good graphic design lies in its language rather than the note of words." Thus, graphic creativity thought plays a vital role in graphic design. In the current global graphic context, people of different countries and areas understand each other and communicate with each other through creative graphic thought and generate the sense of identity.

Innovative development pushes forward the development of human society, being an indispensible part of artistic development. Creative thought brings in the continuous progress of art and design and uninterrupted innovative development is the eternal subject of artistic design. The design method of graphic creativity brings new content into image line drawing, endowing it with new vigor in the new era. The extendability of image line drawing in creative graphic design will constantly enrich manifestation patterns of modern graphs.

\section{RESEARCH ON ANYUE SCULPTURE CARVING IMAGE LINE DRAWING AND GRAPHIC CREATIVITY SUBJECT}

The subject can transfer its attention to operable practice (Specific ways of practice) rather than theory. Through implementation of the subject, people can have a clear understanding of the concept, thought and method. We take the form of a subject, make pointed references in arranging and combining design language of dot, line and face to achieve Anyue sculpture carving's image line drawing, use the method of graphic creativity to constantly intensify the connection between elements and the connotation, put emphasis on images' subjectivity, nonlinearity, time and space, and alternatively use words and graphs to deepen the theme. We decompose and recombine the artistic form of Anyue sculpture carving's image line drawing, and constantly enhance and design to achieve new appearance of image line drawing in order to make Anyue sculpture carving's unique regional culture and art widely spread in the mass audience's mind with new feelings, enrich spiritual connotation and creative manifestation form of image line drawing and extend and carry forward the artistic form of Anyue sculpture carving's image line drawing in modern graphs.

\section{A. Constitution Language}

Modern graphic design adopts the basic two-dimensional design form of dot, line and face and the graph can achieve beauty in form through sequential arrangement of various design elements. The combination law endows the whole picture with form beauty in symmetry and balance, unification and change, rhythm and rhyme and so on. We arrange and combine elements of Anyue sculpture carving's image line drawing through discomposing and reconstruction, create new graphs with aesthetic consciousness, and go beyond regional, racial and cultural difference through strong constitution forms in order to make the author's feeling and emotion accessible to people. For example, the image line drawing themed "Buddhism" demonstrates Anyue sculpture carving's Buddhist statue art. The graph demonstrates various kinds of classic figures of Buddha in Anyue sculpture carvings as extensions of strokes of Chinese character "Fo", showing the charm of figures of Buddha in Anyue sculpture carvings. The author decomposes and rearranges graphs through arrangement of constitution relations of dots and lines in the graph, expressing multiple meanings of classic figures of Buddha in Anyue sculpture carvings and reflecting the author's yearning for Anyue sculpture carvings.

\section{B. Configuration Technique}

Configuration technique refers to the method of using one image structure to demonstrate another image's structure in modern graphic design and it combines and arranges objects which seem to be unrelated superficially and are related in the deep sense to create new images and convey new information. It recombines and rearranges things "similar in meaning or in visual forms" to give people a harmonious and similar feeling, endowing them with new visual and psychological experience. Creative graphs of configuration have the features of intuition, symbolism, readability, suggestibility and aesthetics. We use the design method of creative graphs to reconstruct images in 
image line drawing and to form graphs with deep meanings, and construct new graphs with creativity and aesthetic consciousness, bringing people psychological imagination and emotional experience. Configuration technique is adopted in creative image line drawing of the Apsaras image of Yuanjue rock cave as an example of Anyue sculpture carvings. The carved "Apsaras" in Yuanjue rock cave is placed on the upward side of the head of Bodhisattva holding lotus flowers in her hand in relief. The whole "Apsaras" sculpture boasts elegant and beautiful gesture with floating bands, showing the Apsaras image in Anyue sculpture carving with smooth lines, lightness and elegance. The author uses configuration technique to replace the light and thin ribbon on the body of Apsaras with water flow element and to replace the feature costume of Apsaras with light feature element, symbolizing the Apsaras image smooth as water flow and light as features.

\section{Replacement Method}

Replacement refers to replacing a part of a matter with another thing in order to convey new meanings and achieve new manifestation patterns. It produces a new logic relation of morphological structure and old substance. The information it conveys gives people new connections in mind, intensifying the expressive force of graphs and breaking the limitation of regular thought. The replacement can be done to interior and external elements of images and dash areas of objects. We can constantly find new visual inspiration and make image line drawing spread information in an innovative way through creative replacement of images' external and internal parts. For example, we use the famous black bamboo Avalokitesvara image line drawing in Anyue sculpture carving to replace innovatively. Black bamboo Avalokitesvara is somewhat different from other domestic conventional images of Avalokitesvara straightening her clothes and sitting properly. Black bamboo Avalokitesvara sits sideways with up warped right foot and right hand on the right knee. The whole image is vivid and secular. Even if there is only the outer contour, we can recognize the image of black bamboo Avalokitesvara and feel its extraordinary artistic beauty. We can preserve the outline of black bamboo Avalokitesvara and replace interior elements of it to achieve different graphic effects. For example, we use elements of style and features of Anyue to fill its interior image, bringing different feelings to people. Recollection of Anyue demonstrates that black bamboo Avalokitesvara is rooted on the land of Anyue and reflects the emotional theme of love to Anyue. The imagination creativity revealed on the paper stands vividly and reflects the new visual charm of image line drawing.

\section{Nonlinear Application of Time and Space}

There are many methods to show multi-dimensional images in the two-dimensional way. Since ancient times, people have been observing and demonstrating time and space from various perspectives. As early as in mural painting in tomb chamber of ancient Egypt, side figure frequently appears with front view eyes, demonstrating the spatial display method of using different perspective points to observe. Chinese ancient traditional painting also uses this method frequently. For example, Chinese landscape painting often adopts the method of cavalier perspective. In western painting development of modern times, Picasso, the great master, also adopts this method to paint figures in movement, breaking the limit of time. Image line drawing breaks the spatial limit to combine and arrange image scenes and breaks the time and linearity to combine events of different period. By combining nonlinear contradictory space method with image line drawing, we can not only achieve three-dimensional effect in two-dimensional graphs, but also demonstrate multi-dimensional time and space.

Contradictory space refers to the kind of spatial relation which exists visually, has no existence in three-dimensional space and can be deduced through shift of viewpoint, demonstrating a kind of visionary space. Contradictory space has a multi-viewpoint feature. It creates a brand new inner sense for image line drawing, and demonstrates new visual inspiration and the author's creative consciousness in the contradictory nonlinear time and space, which is well explained in contradictory space image line drawing created for the Apsaras image in Yuanjue rock cave of Anyue sculpture carving. The author constantly changes viewpoints and draws a contradictory three-dimensional triangle on the twodimensional painting with the Apsaras image embedded in it. Seen from various peaks of the three-dimensional triangle, Apsaras exists in each individual viewpoint space. However, seen from the combination perspective, it has no existence in the three-dimensional space. Thus, people have both contradictory and harmonious weird visual experiences.

\section{E. Graphic Characters and Graphic Display of Characters}

Functions of words and graphs are to spread information. Sometimes, words can be taken as graphs to design a painting and graphs can be combined into characters to emphasize the information conveyed. Graphic characters refer to combining graphic elements into characters, which emphasizes on the interestingness of graphic element in characters and brings the function of spreading information in dual ways. This kind of creation method is often used in traditional art, reflecting the unique interestingness and enabling image line drawing to inherit and enrich traditional art consciously. Application of this method requires grasping the harmonization of characters and graphs. Besides graphic characters, there is also the method of graphic display of characters and so on. Graphic display of characters refers to using characters to form a graph. For example, the Custodian King in Pilu cave consists of Diamond Sutra characters. The added character elements make the furious eyes awe-inspiring. The image of the king standing by the sword demonstrates specific connotation details and conveys the author's image theme. For example, various historic spots and folklore characteristic elements of Anyue County are combined with the character of "Yue" to produce images, like the classic reclining Buddha in Anyue sculpture carving and so on. Through reconstruction of various graphs, shapeable elements are smartly integrated into strokes of characters which form the Chinese character "Yue". Hometown features are demonstrated through graphic characters smartly, expressing the author's love for hometown.

\section{CONCLUSION}

Graphic creativity goes with the times. Social development, technological progress and changes in culture aesthetics push 
the concept of modern graph to update and develop constantly. The era features of image line drawing also boost its continuous development, absorption of and integration with new content. Through using methods of design language, configuration, replacement, contradictory space and time, graphic display of characters and graphic characters in graphic creativity to decompose and reconstruct graphs and to expand the innovative thought of image line drawing, image line drawing with Chinese characteristics develops into new national and global features. We combine the method of image line drawing with innovative thoughts of modern graphs to research and practice, and to constantly explore and expand the connotation of image line drawing, enriching manifestation patterns of modern graphic design.

In human's higher level demand design, great emphasis is put on discovering artistic aesthetics and traditional cultural deposits. Traditional art of various places wins a deeper and deeper understanding and more and more emphasis from people. National is global. Excellent traditional art should be inherited and carried forward, needs protection and promotion and requires new methods to explore its inheritance development. Image line drawing integrates design elements and shows its openness in art and creativity. Designers get rid of the stale and bring forth the fresh through exploration and creation, inheriting essence thoughts and cultural deposits of Chinese image line drawing, carrying forward excellent Chinese traditional art, keeping pace with the times and digging out the connotation and extension of image line drawing. It also enriches manifestation patterns of modern graphic design and endows it with a fresh style.

\section{REFERENCES}

[1] Wang Xiangzhi (Song Dynasty): Yu Di Ji Sheng" [M], volume 158 "Puzhou · Fengsu Xingsheng";

[2] Liu Changjiu: China Southwest Grotto Art" [M], Chengdu: Sichuan People's Publishing House, October 1998, p. 19;

[3] Anyue County Annals Compilation Committee of Sichuan Province: "Anyue County Annals" [M], Chengdu: Sichuan People's Publishing House, December 1993;

[4] Xu Shen (Eastern Han Dynasty): Shuowen Jiezi" [M], Beijing: Zhonghua Book Company, 1963;

[5] Yang Kaimin: Application of Image line Drawing in the packaging graph design" [J], "Packaging Engineering", August 2010, Volume 31, No. 18;

[6] [US] Ride] Rudolf - Amnheim: Art and Visual Perception" [M], translated by Teng Shouyao and Zhu Jiangyuan, Chengdu: Sichuan People's Publishing House, March 1998, first edition, p. 133;

[7] Hu Mingfang, Qian Lei: Graphic Design Practice [M], Phoenix publication Media Group, Jiangsu Fine Arts Publishing House, August 2005, first edition; p. 77, p. 92. 